ence of any blood poison. 'There is no regularity in their recurrence. They are brought on by exposure to cold, and exposure to cold only. When the hands or feet get chilled, the patient is seized with shivering, has a general feeling of discomfort, and begins to pass immediately urine more or less darkcoloured from the presence of blood. It may be assumed that there is no organic disease of the kidney, as the urine, except during the attack, is healthy. An alteration in the quality of the blood, or in the state of the bloodvessels, must constitute the immediate precursor of the escape of blood from the kidney. The rapidity with which the effect upon the urine follows the exposure to cold scarcely leaves grounds for supposing that an altered condition of the blood could be set up to occasion it. We have only, therefore, an altered condition of the blood. vessels to fall back upon, and physiology teaches us how rapidly and readily impressions may act through the nervous system, and modify the circulation and nutrition of a part. It is a matter also of the most common observation to witness exposure to cold producing a modification of the circulation in some locality or another. And dispositions of different individuals vary; so that in one the impression of cold, through getting damp feet, for example, shall be followed by a coryza; in another, by bronchitis ; in another, by pleurisy, or, may be, an inflammation of the lungs; in another, peritonitis; in another, inflammation of the kidney; and so on. There are varying degrees of susceptibility appertaining to different parts to be impressed by exposure to cold in different individuals. In the affection that is being commented upon, it may be considered that an unusual susceptibility of the kidney to temporary congestion from exposure to cold happens to exist. The effect, it may be further considered, is determined by the im. pression resulting from the exposure acting through the spinal and sympathetic systems on the muscular coat of the bloodvessels. Taking this view, the principle I acted upon in treatment was, first of all, by suitable coverings or clothing, to diminish the risk of the patient getting chilled, and next to fortify the system by tonies, and render it less impressionable by a narcotic.

Grosvenor-street, July, 1866.

\section{ANEURISM OF THE FEMORAL ARTERY IN HUNTER'S CANAL TREATED BY COMPRESSION. \\ RECOVERY.}

BY ASSISTANT-SURGEON LAURENCE, Ist BatTalion GRENaDIER GUaRDs.

Private J. $\mathrm{P}$ - , aged thirty, a strong, muscular-looking soldier, but of irregular habits, and who has been in hospital frequently with syphilis, was admitted April 4th with pain and stiffness of the right knee-joint. He stated that he first felt uneasiness in the part about a week before admission, and this was followed in two or three days by swelling. The symptoms at the time appeared to be those of articular rheumatism, which, to some extent, subsided under treatment.

On the morning of the 10 th my attention was directed to an enlargement on the inner side of the thigh, about the junction of the middle with the lower third, and on examination forcible pulsation of a distensile and expanding character was detected in the upper part of the swelling. The patient com plained of little or no pain, and the foot and leg were natural in temperature and appearance. He could give no clear account of the origin of the disease, but thinks it not improbable that he might have strained himself in the gymnasium. Aneurism of the femoral artery in Hunter's canal being diagnosed, compression was at once resorted to. A piece of cork, the shape of an ordinary tourniquet pad, was adapted to the broad end of a seven-pound weight, and was applied to the femoral artery as it passed over the pubes. Pressure by means of the double aneurismal compress was tried on three different occasions, but each time had to be discontinued as it caused him too much pain. Digital pressure, varied by that of the weight, was steadily kept up for several days without any apparent beneficial result, but on the morning of the 26 th, on careful examination, no pulsation could be detected. According to the patient's statement it ceased suddenly about three o'clock on the previous day; his attention at the time was called to it as he felt an increased action in the artery above the spot where the pressure was being made, and on placing his hand on the aneurism he found the beating had entirely ceased. From the 26 th up to the present time there has been no return of the pulsation; the tumour has rapidly decreased in size, and is now flat and indistinct.

Remarks. - This case offers some points of interest. The situation of the aneurism being so unusual certainly led one at first to a wrong conclusion, which, perhaps, might have been obviated on a more careful examination. At what time the aneurism might have been detected it is difficult to say, but that it was of rapid formation and quickly enlarged is certain; and for these reasons, as well as the inability of the patient to bear pressure with tourniquets, the case was anything but a promising one, and for days fears were entertained that compression would not be successful. It is of some interest that the man knew when the pulsation in the aneurism had ceased. It stopped suddenly, as if a clot had been detached and thus blocked up the canal that remained open-for previously to this the tumour pulsated strongly, - and thus militates against the idea that it was by gradual deposit of fibrin that the final closure was made. As the statistics of cure of aneurism by compression are somewhat meagre, I may refer to a case of popliteal aneurism I had under my care in 1861 , in which pressure was effectual, the aneurism being perfectly cured; unfortunately, however, the soldier died subsequently of aneurism of the superior mesenteric artery.

June, 1866 .

\section{ON A CASE}

\section{or}

\section{INTENTIONAL, POISONING BY TINCTURE} OF ACONITE; RECOVERY.

By JAMES EASTON, I.R.C.P. EDIN., \&c.

As poisoning by tincture of aconite is of rare occurrence, and as few recover after having taken a large quantity of the more concentrated form of the drug, I deem the particulars of the following case worthy of being recorded in THE LANCET.

M. W- , a servant girl, seventeen years of age, and about five months gone in pregnancy, at nine o'clock A.M. on the 4th of May last, swallowed, with the intention of destroying her life, about three drachms of "Fleming's tincture of aconite"-the contents of a half-ounce phial which was being ad. ministered to cattle in twelve-drop doses three times daily. The girl stated that very soon after having taken the drops she went to a hay-loft and concealed herself for about half an hour, when, becoming alarmed at feeling very severe pains shooting through her head, peculiar tingling sensations throughout her whole body, her arms and limbs also becoming benumbed, a choking sensation and lightness in her head, with partial loss of sight, she went and told her mistress what she had done, who at once administered to her copious draughts of sweet milk (a universal remedy amongst farmers in all cases of poisoning). The girl immediately after this walked, but with a very unsteady gait, to a friend's house about half a mile distant. She was put to bed directly, and very soon afterwards became restless and delirious.

At this stage my attendance was urgently requested; but being at the time about six miles distant, it was nearly eleven o'clock when I arrived. I found her in bed lying on her back, with her limbs much drawn up. Her bedclothes and nightdress were bespattered with mustard and water, which, her attendant said, had been given to her for the purpose of causing vomiting, but which she would not, or, more correctly, could not, swallow. She was conscious; her features were pinched; a dark ring round her eyes; pupils slightly contracted; skin covered with a cold clammy perspiration; breathing hurried and short; pulse 45 , and scarcely perceptible at the wrist; and at intervals she suffered from peculiar choking paroxysms.

As every effort on the part of her attendants to make her vomit failed, I at once administered a full emetic of sulphate of zinc, followed by copious draughts of tepid water, which she drank with considerable difficulty. In about five minutes the emetic began to act, the tepid water meantime being repeated at short intervals. From the large quantity of the ejections the patient must have partaken of a hearty breakfast. Small quantities of diffusible stimulants were now given and repeated at short intervals. Fomentations of mustard and hot water were applied over the heart; warm bread poultices 